\title{
Oesophageal foreign bodies in dogs: factors affecting success of endoscopic retrieval
}

Florence Juvet, Manuel Pinilla, Robert E. Shiel \& Carmel T. Mooney

University Veterinary Hospital

School of Agriculture, Food Science \& Veterinary Medicine

University College Dublin

\section{ABSTRACT}

Oesophageal foreign bodies are common in dogs. Endoscopic removal is a viable treatment option but few studies have assessed the clinical and radiographic features that would be useful in decision-making and prognosis.

Dogs $(n=44)$ with oesophageal foreign bodies presented to the University Veterinary Hospital were assessed. Terriers and West Highland White Terriers were significantly overrepresented $(p<0.0001)$ and in those breeds the foreign body was significantly $(p<0.0001)$ more likely to be located caudal to the heart base. The majority (88.6\%) of foreign bodies were bones or bone fragments.

Group $1(n=30)$ included animals where endoscopic removal was successful and Group 2 ( $n=14$ ) animals where it was unsuccessful or not attempted because of evidence of oesophageal rupture. There was no statistically significant difference in age, sex, body weight, type, location and size of foreign body, recovery rate, short-term complications and long-term outcome between the two groups. Duration of signs prior to presentation and time to spontaneous oral feeding were significantly longer ( $p<0.01$ in each case) in Group 2 (five days and 120 hours, respectively) compared to Group 1 ( 2 days and 24 hours, respectively). Mortality was $11.1 \%$. Long-term follow-up of 29 dogs suggested oesophageal stricture formation manageable by feeding alone in seven (24.1\%) cases.

Terriers appear predisposed to oesophageal foreign bodies. Success of endoscopic removal is adversely affected by duration of signs prior to presentation. Surgical removal negatively influences time to recovery. Stricture formation appears to be a relatively common complication and alternate measures for its prevention should be sought.

KEYWORDS: endoscopic retrieval, oesophageal foreign body, outcome

\section{CORRESPONDING AUTHOR:}

Florence Juvet DMV

University Veterinary Hospital,

School of Agriculture, Food Science \& Veterinary Medicine

University College Dublin

Belfield

Dublin 4

Tel: + 35317166000

Fax: + 35317166023

Irish Veterinary Journal

e-mail: florence.juvet@ucd.ie

\section{INTRODUCTION}

Oesophageal foreign bodies are reasonably common in dogs and can have serious consequences in terms of patient morbidity, mortality and cost (Pollock 1969; Houlton et al. 1985; Spielman et al. 1992; Augusto et al. 2005) Diagnosing the presence of an oesophageal foreign body is usually straight forward, but deciding on whether endoscopic removal is possible may be more challenging. Cases where oeosophageal perforation has already occurred undoubtedly require surgical management (Zimmer 1983). In other cases, endoscopic assessment can be performed with subsequent retrieval per os or foreign body removal after dislodgement into the stomach. If unsuccessful or if unforeseen complications arise, surgical exploration and removal can be attempted. Advantages of endoscopic retrieval include the avoidance of invasive thoracotomy or laparotomy, significant cost reduction and faster time to recovery (Zimmer 1983). 
In addition, endoscopy allows direct visual evaluation of the foreign object and assessment of the extent of oesophageal damage. However, endoscopic removal is not without risk and complications include haemorrhage (Cohn et al. 2003) and oesophageal rupture and its associated complications (pneumomediastinum, pneumothorax, pyothorax). In addition, if unsuccessful and surgery is immediately attempted, the anaesthetic time is markedly increased which may significantly influence the likelihood of post-operative complications (Duncan \& Cohen 1987). Few studies have specifically addressed morbidity and mortality and prognostic factors for successful outcome in dogs (Pearson 1966; Ryan \& Greene 1975; Moore 2001; Sale \& Williams 2006; Leib \& Sartor 2008). It has been shown that the degree of oesophagitis noted after foreign body removal is correlated to the duration and severity of some clinical signs (Rousseau et al. 2007). However, the prognostic utility of other clinical and radiographic features has not been assessed with respect to successful endoscopic retrieval and both short- and long-term outcome.

The objectives of this study were to describe a case series of dogs with oesophageal foreign bodies in order to identify any clinical or radiographic features that may be useful in decision making and prognosis.

\section{MATERIALS AND METHODS}

Medical records of dogs that presented to the University Veterinary Hospital (UVH), University College Dublin with oesophageal foreign bodies between June 2000 and May 2009 were reviewed. Signalment, historical and clinical features and body weight were recorded. The hospital data base (Vetscope Professional, Lawler Developments Ltd.) was interrogated for the total number of all dogs, all terriers and specifically West Highland white terrier (WHWT) dogs presented during the same period.

Thoracic radiographs, when available, were reviewed by a European Diplomate in diagnostic imaging for evidence of mediastinal, pleural or pulmonary pathology. The height and length of the foreign body was measured and recorded. To normalise the size of the foreign body to the size of the dog, the foreign body dimensions were divided by the length of the vertebral body of the sixth thoracic vertebra of each dog. The sixth thoracic vertebra was chosen because of its central position on the radiographs. Endoscopic reports were reviewed for foreign body type and location. The degree of oesophagitis was subjectively classified as mild (superficial erosion), or moderate/ severe (deep ulceration without/with necrosis). Endoscopic removal and, when endoscopic removal was not possible, the type of surgery were also recorded.

Two different flexible endoscopes were used depending on the size of the dogs: a large gastroscope (Olympus GIF Q230, outer diameter 10.5 mm, working length 1030 $\mathrm{mm}$ ) and a small gastroscope (Olympus GIF N230, outer diameter $6 \mathrm{~mm}$, working length $925 \mathrm{~mm}$ ). Various types of forceps or baskets were used for foreign body removal but these were not always specifically recorded. Opting for placement and choosing the type of feeding tube after foreign body removal was at the discretion of the primary clinician.

Dogs were allocated to two groups: Group 1 included animals where endoscopic foreign body removal was successful and Group 2 included animals where endoscopic removal was unsuccessful or where endoscopic removal was not attempted because of prior evidence of oesophageal rupture. Comparisons were made regarding signalment, type and location of foreign body, relative size of the foreign body, duration of signs prior to presentation, mortality rate, time to spontaneous oral feeding, short-term (within one week of foreign body removal) complications and long-term outcome. Long-term outcome was obtained by telephone interview of the owners and ranged from four months to nine years. Complications directly related to feeding tubes are presented separately.

All statistical analyses were performed by computerized software (GraphPad Prism (version 5.02)). Data were analysed for normality by the D'Agostino and Pearson method. Values between groups were compared using the Student's t or Mann Whitney U tests for parametric and non-parametric data, respectively. Categorical data were evaluated by Chi-squared or Fisher's exact test as appropriate. $P$ values less than $0.05(p<0.05)$ were considered statistically significant

\section{RESULTS}

The medical records for 45 dogs treated for oesophageal foreign bodies during the study period were reviewed. One case was excluded because the foreign body was a linear intestinal foreign body located from the oesophagus to the small intestine. The foreign body was successfully removed from the oesophagus endoscopically in 30 (68.2\%) cases of which 26 (86.7\%) were retrieved per os and four (13.3\%) were pushed into the stomach. Endoscopic foreign body removal was unsuccessful in $11(25.0 \%)$ cases. It was not attempted in three (6.8\%) animals because of evidence of oesophageal rupture, identified on radiographs in 2 cases and on inflation at endoscopy in one case. Surgical procedures necessary for foreign body removal were cervical oesophagotomy $(n=3)$, thoracotomy $(n=4)$ and laparotomy and gastrotomy $(n=7)$.

The mean $( \pm \mathrm{sd})$ age was $5.7( \pm 3.9)$ (range, 0.2 - 15.0) years. The median body weight was 7.3 (range, $1.8-$ 56.0) kg. Group 1 consisted of 14 males and 16 females with a mean age of $6.2( \pm 4.1)$ years and a median weight of $7.05 \mathrm{~kg}$. Group 2 consisted of six males and eight females with a mean age of $4.9( \pm 3.4)$ years and a median weight of $7.8 \mathrm{~kg}$. There was no statistically significant difference in age, sex or body weight between the two groups.

Overall, 15 breeds were represented, including West Highland White Terriers (WHWT) $(n=19)$, Shih Tzu $(n=$ $5)$, Yorkshire Terrier $(n=4)$, Cavalier King Charles $(n=2)$, Jack Russell Terrier $(n=3)$, pomeranian $(n=2)$ and one of each of the following: Staffordshire Bull, Tibetan, Wheaten and Cairn Terriers, Collie, Bull Mastiff, mixed-breed terrier, 
Labrador-cross and English Bull Dog. Over the same time period, 29,235 dogs were presented to the UVH including 4,567 terriers and 813 WHWT. There were significantly $(p<$ 0.001 , in each case) more WHWT and terriers in the dogs presenting with oesophageal foreign bodies than those presenting for other reasons.

The presenting clinical signs were similar in nature and frequency in both groups and included regurgitation/ vomiting (70\% (Group 1) and 93\% (Group 2)), inappetence/ anorexia (30\% (Group 1) and 60\% (Group 2)), lethargy/ dullness (30\% (Group 1) and 20\% (Group 2)) and retching (20\% (Group 1) and 13\% (Group 2)). The duration of signs prior to foreign body removal ranged from two to 42 days and the median time was significantly longer in Group 2 (five days) compared with Group 1 (two days) ( $p<0.01$ ). Physical examination was unrewarding in most cases apart from when the foreign body was located in the cervical oesophagus (palpable in one case).

Table 1 - Comparison of Group 1 (successful endoscopic removal) and Group 2 (unsuccessful endoscopic removal) dogs with regard to type and location of foreign body, size of foreign body, mucosal damage, short term complications, time to spontaneous oral feeding and long-term clinical outcome.

Type of Foreign Body

\begin{tabular}{|l|l|l|}
\hline Bone & $26(86.7 \%)$ & $13(93.3 \%)$ \\
\hline Soft & $4(13.3 \%)$ & - \\
\hline Stick & - & $1(7.1 \%)$ \\
\hline & \multicolumn{2}{|c|}{ Location of Foreign Body } \\
\hline Cervical oesophagus & $3(10 \%)$ & $3(21.4 \%)$ \\
\hline Heart base & $6(20 \%)$ & $2(14.3 \%)$ \\
\hline Caudal to heart base & $21(70 \%)$ & $9(64.3 \%)$ \\
\hline Normalised mean length $(\mathrm{cm})^{*}$ & 3.3 & 3.3 \\
\hline Normalised mean height $(\mathrm{cm})^{*}$ & 2.2 & 1.9 \\
\hline
\end{tabular}

Degree of oesophagitis

\begin{tabular}{|l|l|l|}
\hline Mild & $16(53.3 \%)$ & $4(28.6 \%)$ \\
\hline Moderate/severe & $12(40.0 \%)$ & $9(64.3 \%)$ \\
\hline Not specified & $2(6.7 \%)$ & $1(7.1 \%)$ \\
\hline \multicolumn{2}{|c|}{ Short-term complications } \\
\hline Mild & $3(10 \%)$ & $2(14.3 \%)$ \\
\hline Severe & $5(16.7 \%)$ & $3(21.4 \%)$ \\
\hline $\begin{array}{l}\text { Median time to spontaneous oral } \\
\text { feeding (hours) }\end{array}$ & $24(12-240)$ & $120(24-360)$ \\
\hline Long Term complications** & 5 & 3 \\
\hline
\end{tabular}

\section{FB - foreign body}

*Measurement were only assessed in 29 cases

** Only 21 of Group 1 and eight of Group 2 dogs were available for long-term, follow-up
Details of foreign body type, location and size, degree of oesophagitis, time to spontaneous oral feeding and shortand long-term complications are presented in Table $\mathbf{1}$.

The foreign body was located caudal to the heart base in $30(68.2 \%)$, and either at the heart base $(n=8(18.2 \%))$ or in the cervical oesophagus $(n=6(13.6 \%))$ in 14 cases. There was no significant difference in location between the two groups. In the 31 terrier dogs, 26 (83.9\%) of the foreign bodies were located caudal to the heart base compared with only 4 (28.6\%) of the remaining 14 dogs. This difference was statistically significant $(p<0.001)$. Thirty nine (86.6\%) foreign bodies were bones, including 23 chops/vertebrae (12 lamb, 6 pork and 5 unidentified species), 2 chicken bones, 1 turkey bone, 1 beef bone and 12 other bone types for which species was not reported. There was one each of the following: commercial dried pig ear, piece of cooked chicken breast, potato, piece of silicone, and stick.

Radiographs were available for review in 33 cases. The foreign body was seen in all cases with the exception of one dog in which a stick foreign body was not visible. Changes secondary to the foreign body were present in two cases (pleural effusion, aspiration pneumonia and mediastinitis in one and free gas in the soft tissues of the neck in the other). Height and length of the foreign body could be determined in 29 cases, including 18 cases of Group 1 and 11 cases of Group 2. There was no statistical difference in foreign body size between the two groups or between the different breed (terriers versus non-terriers) groups.

The degree of oesophagitis and the incidence of mild or severe short-term complications were not statistically different between the two groups. Mild short-term complications included isolated episodes of vomiting (three cases of group 1 and one case of group 2) and prolonged inappetence. Severe complications included aspiration pneumonia ( $n=3$, all from Group 1 ) oesophageal rupture or necrosis ( $n=3,1$ of Group 1 and 2 of Group 2 ), haemorrhage ( $n=1$ from Group 2) and formation of a diverticulum ( $n=1$, from Group 1 ). Two dogs in each group died (extensive oesophageal mucosal necrosis noted on endoscopy 3 and 5 days after initial presentation (one each from Group 1 and 2), aspiration pneumonia (Group 1) and intra-operative haemorrhage (Group 2)). The overall mortality rate was $6.7 \%$, and $14.3 \%$ for Group 1 and 2, respectively. This difference was not statistically significant. The time to spontaneous oral feeding (median) was significantly shorter in Group 1 (24h) compared to Group 2 (120h) $(\mathrm{p}<0.01)$.

The long-term outcome was known for 29 dogs including 21 in Group 1 and eight in Group 2. Amongst these dogs, 21 (72.4\%) dogs had made a full recovery and were fed their usual diet. One (4.8\%) dog (Group 1) developed a diverticulum 10 days post foreign body removal and died during its surgical correction. Seven (24.1\%) dogs were considered to have major (inability to eat unmashed food) long-term complications including three of Group 2 (37.5\%) and four of Group 1 (19.0\%). There was no 
statistically significant difference in the number of longterm complications between the two groups. A definitive diagnosis was not achieved in these cases. Oesophageal stricture formation was considered likely although further investigation and intervention (balloon dilation or bougienage) were not performed. The duration of signs prior to removal and the size of the foreign bodies were not statistically different in the seven dogs with long-term complications as compared to the 21 dogs that survived with no known long-term complications.

\section{DISCUSSION}

The present retrospective study confirms some findings of other studies and offers some new insights into this disorder for the small animal practitioner. Specifically, investigating those factors that influence the outcome for endoscopic foreign body retrieval has practical implications regarding estimated anaesthetic time and likelihood of surgical intervention.

The type of dogs presented (middle-aged, small breeds), and their clinical signs were similar to previous reports (Pearson 1966; Ryan \& Greene 1975; Houlton et al. 1985; Spielman et al. 1992). In addition, they did not differ between the two groups presented in the study and signalment or clinical features provided no insight into success or otherwise of endoscopic foreign body retrieval. Several other studies have suggested terrier breeds as being over-represented (Pearson 1966; Houlton et al. 1985; Spielman et al. 1992), although in one study poodles were overrepresented (Ryan \& Greene 1975), suggesting that breed predisposition may be more representative of the general dog population. However, in the present study there was a definite breed predisposition for terrier breeds and WHWT compared to the general hospital population interrogated over the same time period. As a consequence, while other disorders may give rise to similar clinical signs of acute regurgitation and retching, oesophageal foreign bodies should be given greater priority in terriers and WHWT in particular.

In dogs, bones or bone fragments are the most common cause of oesophageal obstruction ranging from $47 \%$ to $100 \%$ in reported studies (Ryan \& Greene 1975; Houlton et al. 1985; Moore 2001; Rousseau et al. 2007). Similarly, the majority of foreign bodies were bones in the present study. Foreign bodies typically lodge in the oesophagus where there is physiologic narrowing including the pharynx, thoracic inlet, heart base and distal oesophagus. All regions except for the pharynx were represented in the present study and overall the most commonly affected area was the distal oesophagus caudal to the heart base. Interestingly, while location did not affect outcome, it was different between the terriers and non-terriers being significantly more commonly found caudal to the heart base in the former group. It is unclear why terriers are over-represented or why the location varies. Behavioural differences have been speculated with little scientific foundation (Moore 2001). A second possible explanation is that the sites of reduced oesophageal diameter may be different between breeds and this concept may be supported by the different locations found in the present study. However, another possibility is that terriers are predisposed to oesophageal motility disorders and gastro-oesophageal reflux (GER), as reported previously (Bexfield et al. 2006). A motility disorder increases the risk of oesophageal foreign bodies normally conducted to the stomach in other breeds. In addition, GER could predispose them to slight stricture formation of the distal oesophagus accounting for the different location as reported here. However this is largely speculative and motility disorders previously reported in terriers have only been demonstrated in young dogs, younger than the cases presented in the current study.

Radiographs were available for retrospective study in the majority of cases. Not surprisingly given that most foreign bodies were bones, most were visible radiographically. In order to account for differences in breed size, the dimensions of the foreign bodies were normalised to the individual animal. However, the size of the foreign bodydid not influence outcome or the incidence of longterm complications and should not be used as a reason to avoid endoscopic retrieval. Despite this, radiographic assessment is still valuable as it provides important information on location and on changes that can occur secondary to the foreign body (perforation, aspiration pneumonia).

The overall success rate of just over $65 \%$ for endoscopic retrieval or dislodgement was lower in this study compared to rates of between $73 \%$ and $87 \%$ reported elsewhere (Spielman et al. 1992; Rousseau et al. 2007). This may reflect the fact that duration of clinical signs prior to presentation was longer than in most previous reports combined with a different emphasis in this study as an attempt was being made to better define factors that could predict outcome. Specifically animals in which endoscopic retrieval was not attempted because of known complications (e.g. oesophageal rupture) were also included. If such animals were excluded, the overall success rate was approximately $73 \%$ and therefore more comparable to the previous studies. The recovery rate and incidence and type of short-term complications is also similar in this and other studies (Spielman et al. 1992; Elliott et al. 2000; Rousseau et al. 2007). Overall there was a relatively low rate of short-term complications together with a low mortality rate of just over $10 \%$ in accordance with previous studies (Spielman et al. 1992; Hotston Moore 2001; Rousseau et al. 2007), while other studies have reported mortality rates of 15.2-26\% (Ryan \& Greene 1975; Pearson 1966; Leib \& Sartor 2008). However, dogs in which surgical intervention was required took significantly longer before spontaneously feeding resulting in more prolonged hospitalisation and increased costs.

In this study the degree of oesophagitis did not influence the success or otherwise of endoscopic retrieval or dislodgement. However, clinical signs were present for longer in those dogs in which endoscopic removal was not 
successful. By contrast in another study, the duration of clinical signs prior to presentation was significantly shorter for dogs with mild oesophagitis compared to those with moderate-to-severe oesphagitis (Rousseau et al. 2007). This difference may be explained by the way in which oesophageal damage was assessed in the latter paper using the Savary-Miller classification which puts more emphasis on the confluence of erosions rather than the more subjective classification used here. Nevertheless it is reasonable to suggest that the longer the foreign body is present the more likely an alternative to endoscopy is required for removal. As a consequence, such cases should be managed early in the day with appropriate preparation for possible surgical intervention.

Of the 28 survivors for which long-term outcome was known, approximately one quarter were suspected to have oesophageal stricture, based on the owners reports of regurgitation of any food not mashed beforehand. Few studies have clearly reported the rate of stricture formation but it seems that in older reports stricture formation was rare (Hotston Moore 2001; Spielman 1992) while in more recent studies a rather high incidence of complications was identified on long-term follow-up. In a recent retrospective study of oesophageal obstruction caused by a dental chew treat, oesophageal stricture developed in 6 of 25 dogs (24.0\%) that survived initial hospitalisation (Lieb \& Sartor 2008). In another retrospective study of 60 cases, mild stricture formation was reported 9-21 days post-removal in 5 of 17 (29.4\%) dogs for which outcome was available and this only occurred in dogs previously classified as having moderate-to-severe oesophagitis (Rousseau et al. 2007). While intuitively, stricture formation would be considered more likely associated with more severe damage to the oesophagus, this was not the case in the present study. Again, the subjective way the oesophageal damage was classified may be responsible for the difference in the studies. Due to the retrospective nature of this study, a classification based on circumference damage was not possible but may correlate better to the rate of stricture formation. However, the fact that the rate of stricture formation was high in a previous report on dental chews (Leib \& Sartor 2008) and developed in cases presented here with soft foreign bodies and in cases where damage to the oesophageal mucosa was judged mild, suggests that stricture formation is a possible consequence of any oesophageal foreign body and may relate more to the extent of contact rather than its oesophageal penetration. To prevent stricture formation, the use of topical and/ or systemic antacids is standard (Zimmer 1983; Sellon $\&$ Willard 2003). Administration of antibiotics along with dietary restriction is also traditionally recommended. Withholding food and water, providing parenteral or gastric nutrition and hydration, is thought to minimize trauma to the mucosa and reduce fibroblastic reaction responsible for stricture formation (Zimmer 1983). All of these measures are routinely implemented in the UVH but obviously do not decrease the risk completely and other preventive measures should be investigated. Although withholding food per os post-removal is usually recommended for preventing oesophageal strictures, it potentially could favour stricture formation by preventing natural stretch with larger food particles. Studies directly comparing dogs fed a normal diet and those fed liquid food post-foreign body removal have not yet been published. In humans, the use of mitomycin-C an anthracycline, after oesophageal dilatation has been shown to be effective in reducing the rate of stricture reformation (Kumar \& Bhatnagar 2005). Research in rats, after standardised trauma to the oesophagus, show promising results with the antimetabolic agent halofuginone when topically applied (Ertug et al. 2009). Similar research is lacking in dogs and cats.

\section{CONCLUSION}

This retrospective study of oesophageal foreign bodies confirms the predisposition of terriers and WHWT in particular and demonstrates a different location for their foreign bodies. Unfortunately neither clinical nor radiographic findings were helpful in predicting the success or otherwise of endoscopic removal. However the longer the duration of clinical signs, the more likely alternative surgical intervention is required. Dysphagia/regurgitation, suggestive of oesophageal stricture formation was a relatively common long-term complication in this study. Further studies are required to investigate measures to prevent stricture formation following the treatment of oesophageal foreign bodies in dogs.

\section{REFERENCES}

Bexfield, N. H., Watson P.J., Herrtage M.E. (2006) Esophageal dysmotility in young dogs. Journal of Veterinary Internal Medicine 20, 1314-1318.

Cohn, L. A., M. R. Stoll, Branson, K.R. et al. (2003) Fatal hemothorax following management of an esophageal foreign body. Journal of the American Animal Hospital Association 39, 251-256.

Duncan, P. G., Cohen M.M. (1987) Post-operative complications: factors of significance to anaesthetic practice. Canadian Journal of Anaesthesia 34, 2-8.

Elliott, D. A., Riel D.L., Rogers Q.R. (2000) Complications and outcomes associated with use of gastrostomy tubes for nutritional management of dogs with renal failure: 56 cases (1994-1999). Journal of the American Veterinary Medical Association 217, 1337-1342.

Ertug, E., Cekin, I., Cincik, H.,et al. (2009) Effectiveness of topically applied halofuginone in management of subglottic stenosis in rats. Otolaryngology - Head and Neck Surgery 140, 720-723.

Houlton, J. E. F., Herrtage, M.E., Taylor, et al. (1985) Thoracic oesophageal foreign bodies in the dog: a review of 90 cases. Journal of Small Animal Practice 26, 521-536.

Kumar, A., Bhatnagar V. (2005) Topical application of mitomycin-C in corrosive esophageal strictures. Journal of the Indian Association of Pediatric Surgery 10, 25-27.

Leib, M. S., Sartor L.L. (2008) Esophageal foreign body 
obstruction caused by a dental chew treat in 31 dogs (2000-2006). Journal of the American Veterinary Medical Association 232, 1021-1025.

Moore, A. H. (2001) Removal of oesophageal foreign bodies in dogs: use of the fluoroscopic method and outcome. Journal of Small Animal Practice 42, 227230.

Pearson, H. (1966) Symposium on conditions of the canine oesophagus: foreign bodies in the oesophagus. Journal of Small Animal Practice 7, 107-116.

Pollock, S. (1969) Esophageal foreign body in the dog. Journal of the American Veterinary Medical Association 155, 531-534.

Rousseau, A., Prittie, J., Broussard, J. D., et al. (2007) Incidence and characterization of esophagitis following esophageal foreign body removal in dogs: 60 cases (1999-2003). Journal of Veterinary Emergency and Critical Care 17, 159-163.

Ryan, W. W., Greene, R.W (1975) The conservative management of esophageal foreign bodies and their complications: a review of 66 cases in dogs and cats. Journal of the American Animal Hospital Association 11, 243-247.

Sale, C. S., Williams, J. M. (2006) Results of transthoracic esophagotomy retrieval of esophageal foreign body obstructions in dogs: 14 cases (2000-2004). Journal of the American Animal Hospital Association 42, 450456.

Sellon, R. K., Willard, M. D. (2003) Esophagitis and esophageal strictures. Veterinary Clinics of North America: Small Animal Practice 33, 945-967.

Spielman, B. L., Shaker, E. H., Garvey, M. S. (1992) Esophageal foreign body in dogs: a retrospective study of 23 cases. Journal of the American Animal Hospital Association 28, 570-574.

Zimmer, J. F. (1984) Canine esophageal foreign bodies: endoscopic, surgical, and medical management. Journal of the American Animal Hospital Association 20, 669-677. 